\title{
Study of a Hybrid Algorithm for Indoor Positioning
}

\author{
Hee-Yong Kang ${ }^{1}$, Ji-Na Lee ${ }^{2}$, Hyungwoo Park ${ }^{3}$, Myung-Jin Bae ${ }^{4}$ \\ and Jong-Bae $\mathrm{Kim}^{5 *}$ \\ ${ }^{1,2}$ Dept. IT Policy and Management, Soongsil University, Sangdo-dong, \\ Dongjak-gu, Seoul, Korea \\ ${ }^{3,4}$ Dept. Information \& Technology Soongsil University, Sangdo-ro 369, \\ Dongjak-gu, Seoul, Republic of Korea \\ ${ }^{5 *}$ Graduate School of Software, Soongsil University, Sangdo-dong, \\ Dongjak-gu, Seoul 06978, Korea \\ 1hykang07@naver.com, ${ }^{2}$ ppjina@hanmail.net, ${ }^{3}$ pphw@ssu.ac.kr, \\ ${ }^{4}$ mjbae@ssu.ac.kr, ${ }^{5 *} k j b 123 @ s s u . a c . k r$
}

\begin{abstract}
Today almost everyone has a device with positioning capabilities such as a smartphone, tablet, GPS tracking device or watch with built-in GPS. Positioning systems have played a major role in people's lives since the required technology became publicly available in the 1990's. In recent year, Indoor positioning technology has become commercially available in various forms and with varying levels of quality, unlikely the outdoor positioning system that is enabled by GPS satellite technology, there is still no perfectly proven indoor positioning method that satisfies all the required purposes. Each of the indoor positioning technologies has both strengths and weaknesses. Indoor positioning technologies differ in terms of accuracy, costs and efforts, but have improved quickly in the last couple of years. Bluetooth Low Energy is a universal low-power wireless standard that makes easy to connect a wide range of products including smart phones or tablets. This paper proposes an algorithm that enhances the accuracy and efficiency of an indoor positioning system by integrating the BLE positioning circle technique with the PDR technique using the IMU data of terminals. This study shows that the proposed technique improves the position accuracy to within $2.1 \mathrm{~m}$ and enables its application to indoor navigation systems for very large spaces like airports and stadiums.
\end{abstract}

Keywords: BLE Beacon, Positioning Circle Technique. Hybrid BLE and PDR, Indoor Positioning

\section{Introduction}

As smart phones are rapidly spreading these days, research into to providing locationbased services for indoor spaces has been increasing. Existing indoor positioning technologies typically include Ultra-Wide Band using impulses and Bluetooth which use a lot of bandwidth, creating EMI (Electro Magnetic Interference) in legacy systems such as indoor electrical and wireless devices. Furthermore, owing to its short communication range, Beacon indoor positioning does not guarantee positioning accuracy, making it a suboptimal choice considering its high installation and maintenance costs $[6,10,11]$.

BLE (Bluetooth Low Energy), an alternative technology that was developed to solve these problems, is used as a technology for IPS (Indoor Positioning Systems) together with a trilateration technique, a fingerprinting technique, and a Pedestrian Dead

Received (March 16, 2018), Review Result (June 15, 2018), Accepted (June 20, 2018)

* Corresponding Author 
Reckoning (PDR) technique for sensor data of an IMU (Interactive Measurement Unit)[3][6].

However, each of these techniques has both strengths and weaknesses at the. UWB, which has the highest precision, wide range, and dynamic positioning capability, requires infrastructure such as transmitters and receivers as Bluetooth, and in order to use the fingerprinting technique to complement the RSSI of the WiFi, it requires reference point mapping by dividing the entire space.

In this paper, we propose a positioning circle which addresses the low accuracy - i.e., large positional deviation - issue a weakness of the existing BLE based RSSI method, and an algorithm which improves the accuracy and efficiency of indoor positioning system by converging the PDR method using the IMU data of mobile devices.

\section{Related Works}

\subsection{Wi-Fi}

Wi-Fi technology is the most common technology embedded in most mobile devices because the algorithm is simple and is not technically challenging. Triangulation and fingerprinting techniques are used to estimate position. Triangulation is a typical technique of indoor position estimation, and involves measuring the RSSI values from three or more access points (APs) and converting the measured values to distances to estimate the position.

However, the indoor position estimations show lower accuracy mainly due to multipath propagation phenomena such as attenuation, reflection, and refraction of wireless signals caused by various obstacles such as walls, pillars, and moving crowds in the indoor space, making it difficult to create an intersection for more than three APs.

As an alternative to overcome this problem, a fingerprinting technique is applied. This technique divides the indoor space into small cells, collects RSSI values directly from each cell, and constructs a radio map by converting them into a database [4]. This technique is used to estimate a user's location through comparing received RSSI values with the database and selecting the cells of the most similar signal pattern. It has a database that reflects the characteristics of the space, so it is much more accurate than the trigonometric method.

While Wi-Fi has the advantage of sharing the wireless network infrastructure and thus does not require the installation of an additional hardware infrastructure, it involves the cost of calibrating a radio map. Also, additional maintenance costs are incurred in the event of AP replacement or movement, requiring resurveys. Its commercialization requires that a number of problems be addressed, including survey cost, positioning performance, battery consumption, RSSI heterogeneity between devices, error estimation, Kalman filter and map matching. Recently the Wi-Fi SLAM (Wi-Fi Simultaneous Localization and Mapping) technique was introduced, which enables an operator to automatically configure the radio map simply by walking around the indoor space, making it faster than the conventional method, with a cost reduction of 10 to $20 \%$.

\subsection{BLE}

BLE is a typical technology applied to indoor positioning, as most smart devices support BLE. It is one of the most recently emergent technologies and has become an industry standard available on most devices today. BLE beacons or iBeacons are inexpensive, small, provide a long battery life and do not require an external energy source. The device detects the signal from a beacon and can roughly calculate the distance to the beacon and hence estimate the location. 
BLE beacon based indoor positioning is a proximity technique, while AOA (Angle of Arrival) and trilateration are highly accurate indoor positioning techniques based on BLE technique.

Proximity techniques approximate a device's position according to a beacon's receiving device signal. When the device is in close proximity to a beacon which generates a very weak signal, it is assumed that the user is in a beacon position, as Apple's iBeacon is. This provides a very simple method and supports the miniaturization of Beacon and long-term battery operation, so it can be attached to various locations in the indoor space, providing variety of services to users. However, due to the short signal coverage of Beacon (Beacon), a number of beacons must be installed to enable estimation of indoor positioning in entire space of airports or large shopping malls.

The AOA technique uses a specially designed receiver with a high-precision indoor positioning technique and has an error range of less than $1 \mathrm{~m}$. Its receiver is semicircular and has several directional antennas on the surface, so that the device's signal is received only on a specific antenna. It calculates the position of the user based on the angle of the received signal and the position of the receiver. One receiver installation allows $2 \mathrm{D}$ positioning in a certain range of space, providing relatively high accuracy and enabling 3D positioning by installing two or more, but the technique requires dedicated receiver installation, and it does not the Bluetooth standard applied, and thus cannot be used with smartphones yet.

The trilateration technique uses the characteristics of low-powered BLE as a technique to measure and locate the respective signal intensity (RSSI). BLE is cheap and easier to install and repair than $\mathrm{Wi}-\mathrm{Fi}$ as it only uses beacon. Its advantage is more precise positioning within a certain space. Recently, studies and implementations of indoor positioning technology using BLE have been drawing attention. On the other hand, since the protocol of BLE beacon is not dedicated to positioning, it is necessary to set the transmission period and decide the number of installations to prevent collision between signals caused by an increasing number of beacons and a short transmission period for positioning [5]. In addition, it is essential to filter out RSSI values received from signal drop out.

\subsection{Inertial Measurement Sensors}

A gyroscope is a device for measuring or maintaining orientation, based on the principles of conservation of angular momentum. Most smartphones have a built-in gyroscope and compass. The device can use this information in addition to the other technologies mentioned for even more precise positioning.

\section{BLE Beacon Based Precise Indoor Position}

In this study, we propose an algorithm that applies the trilateration technique to remedy the weakness of the existing GPS positioning method. Trilateration is a technique of estimating a user position by measuring RSSI intensity between three or more AP nodes and a mobile device. Of the indoor positioning techniques, trilateration has advantages in that it can figure out user location with only a few APs, and is also easy to install and repair.

The signal information obtained from IMU such as a three-axis accelerometer, a gyroscope, a magnetometer and a barometer is utilized to improve the accuracy of indoor positioning. In other words, Pedestrian Dead Reckoning using the function of the inertial sensor mounted on a smartphone is applied as a means of error correction to increase the accuracy of indoor position estimation.

The combined positioning system consists of a low and high precise integration method. Low-precise integration estimates a position with each signal from APs and assumes the result as a reliable and accurate position. High-precise integration estimates a 
position using multiple signal values from the position estimation step [6]. The lowprecise integration method is simple to implement but its accuracy is low, high-precision integration's accuracy is high, but its implementation is complicated and a new proprietary algorithm for complex positioning must thus be devised.

BLE Beacons and App are required to provide BLE indoor positioning. BLE Beacons are small devices and can be easily mounted on walls. Their mission is to help Smartphones determine the location. BLE beacons use battery power and consume very little energy. Newer beacons can run for years on a single battery charge. An app for Smartphone or tablet is required to provide navigation and location triggered content service. We usually choose between generic and branded apps.

In this paper, we propose a hybrid indoor positioning algorithm that combines Bluetooth Low Energy-based technique and PDR technique for more precision.

\subsection{Bluetooth Low Energy-based Technique}

Figure 1 below shows the whole structure of BLE based indoor positioning process. A mobile device downloads each beacon's location information from the server and then analyzes the signal strength from each BLE beacon to perform indoor positioning.

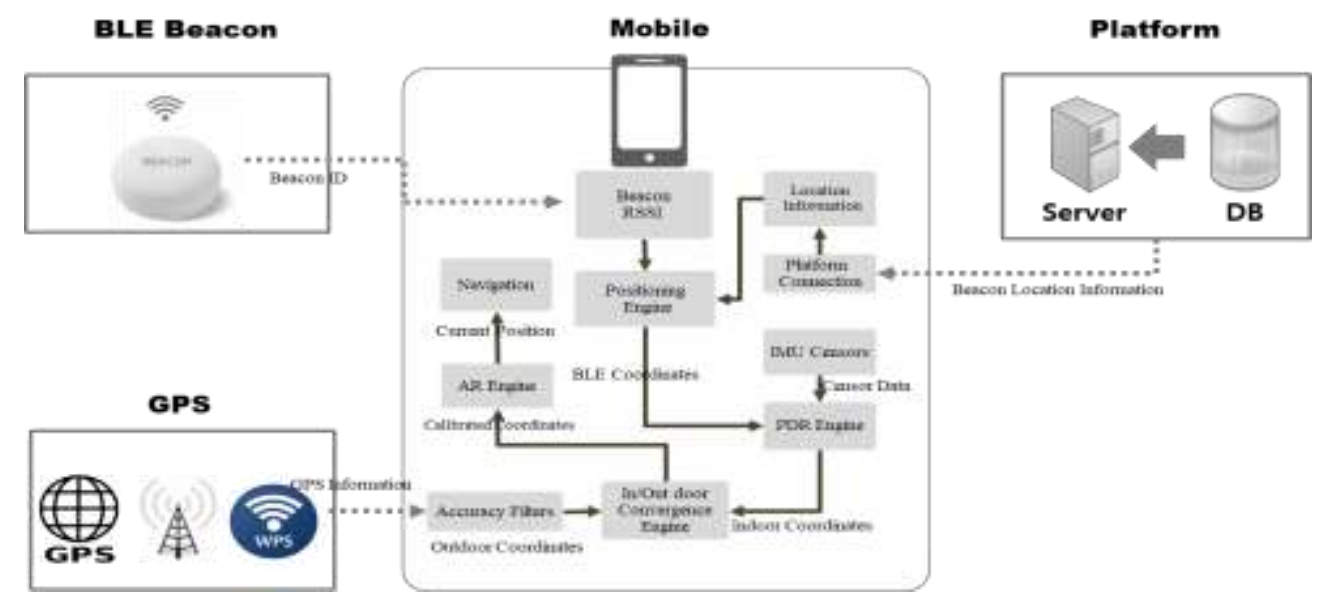

Figure 1. Structure of Hybrid Indoor Positioning System

The indoor positioning algorithm using BLE devised in this study proceeds in the following order.

First, the mobile device downloads the BLE beacons installation map information from the server. The information contains each installed beacons' ID, latitude, longitude coordinate, and others.

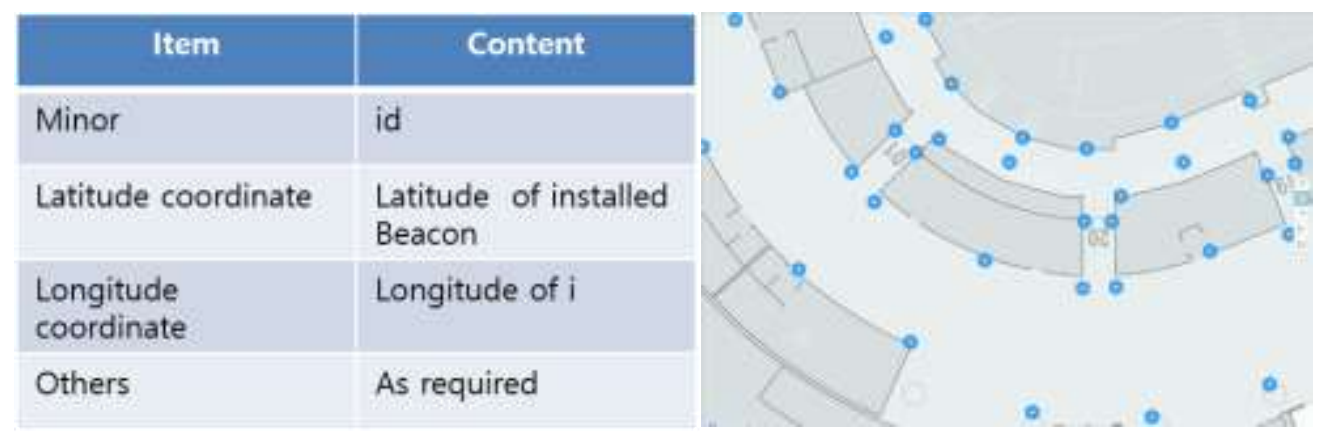

Figure 2. BLE Beacon Map Information 
Second, the mobile device scans the UUID, RSSI, minor and major values from the BLE beacons utilizing its built-in iOS or Android BLE scan service.

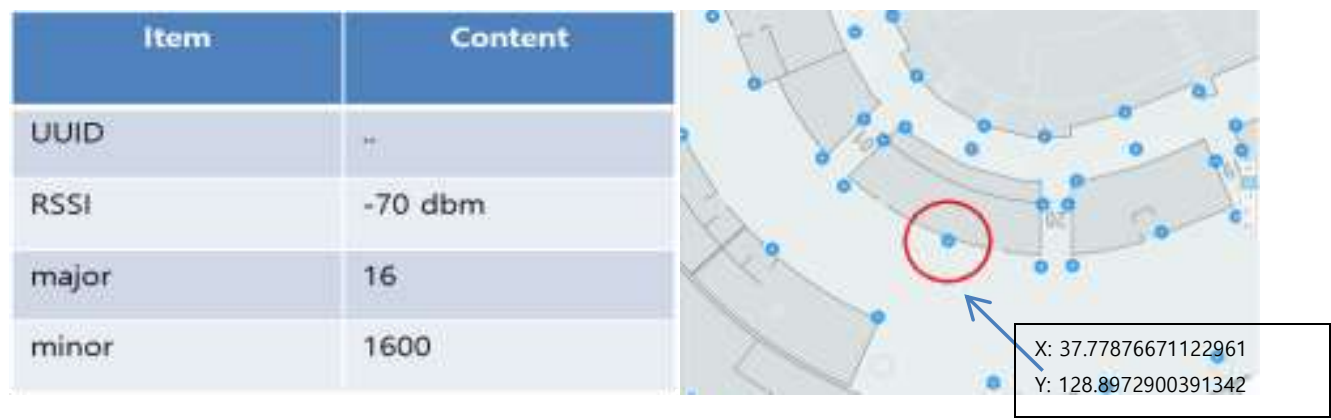

Figure 3. Scanned Beacon Information and Coordinates

The scanned beacon's minor values are compared with the downloaded beacon installation information list to obtain the $\mathrm{x}$ and $\mathrm{y}$ coordinates of the beacon. The coordinates of all scanned beacons are obtained through the same procedure.

In the third step, which is a pre-step for positioning circle generation to improve the of accuracy indoor positioning, the coordinates of all scanned beacons are found and compared with information of the BLE beacon map. The beacon information, such as its minor value, RSSI value, $\mathrm{x}$, y coordinates which are scanned in real time for indoor positioning is stored in the beacon data scan list.

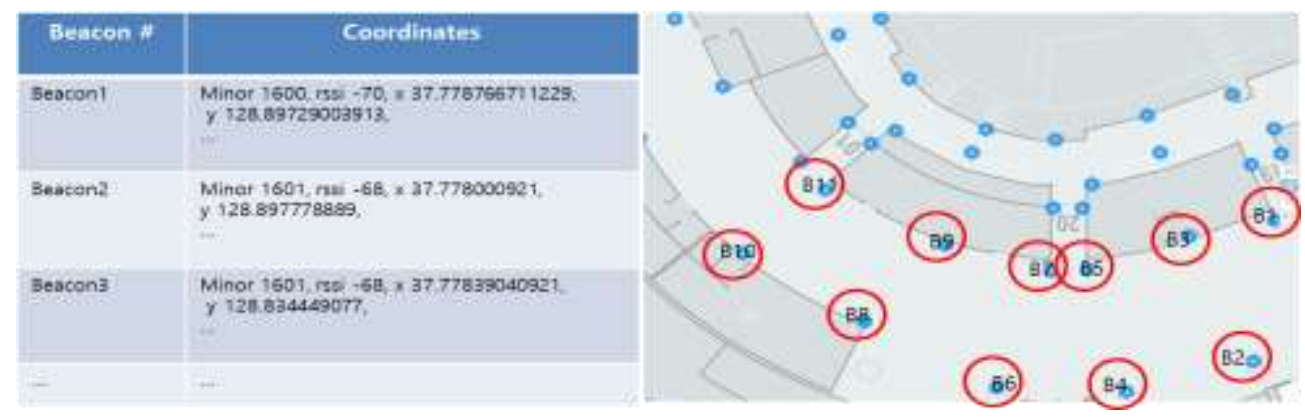

Figure 4. Scanned Beacon's Location Information

For the process of generating the BLE Beacon scan list, we applied pre-processes of RSSI value as a first step. The RSSI value of each beacon received in the process of scanning the RSSI of BLE Beacon is highly dispersed by multipath fading. The Scan List is created by calculating the coordinates and distance of each BLE Beacon through an RSSI pre-processing process in order to distinguish only useful RSSI values by removing outliers and calculating a mean and standard deviation with moving average. Depending on whether a pre-processed RSSI value is new or not, it is decided either to add to or update in the Scan list. The average value of the remaining RSSI is calculated and used as a pre-processed value in the next step.

The next is the Kalman filtering process. Distance between BLE beacon and mobile device is calculated using the pre-processed RSSI value from the previous step such as RSSI value filtering or/and Parsing.

For more precise distance calculation, the RSSI value calculated through the preprocessing process is calibrated by applying the actual distance calculation equation (1) of the Kalman filtering algorithm, where rssi_p is the pre-processed RSSI value and rssi-cali is one-meter distance based calibrated value [2][7]. 


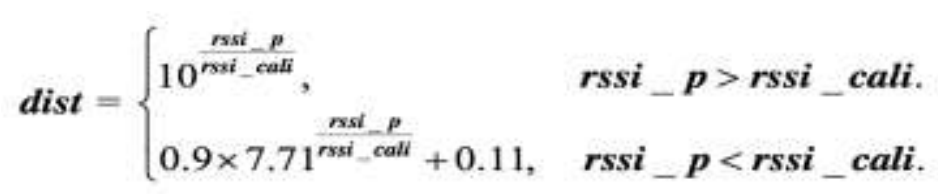

The Kalman filtering algorithm of Eq. (1) is applied to the pre-processing process to more accurately calculate actual distance between the mobile device and BLE beacon.

The final of the third step is a Trilateration process. In order to reduce the deviation from the actual distance even after Kalman filtering, we applied the trilateration technique, which calculates the distance of three BLE beacons closest to the mobile device to determine the device location. The distance is calculated using a standard RSSI attenuation model based on the pre-computed distance around each.

To reduce deviation from the actual distance by Kalman filtering, we apply the trilateration technique, which calculates the distance of three BLE beacons closest to the mobile device and its location. The distance is calculated using standard RSSI attenuation model (2) based on the pre-computed distance around each.

$$
d_{m}=10^{\frac{R S S l-A}{-10 \cdot n}}
$$

The algorithm of these three processes to generate the scan list, which will be used for calculation between mobile device and BLE beacon is as shown in Figure 5 below.

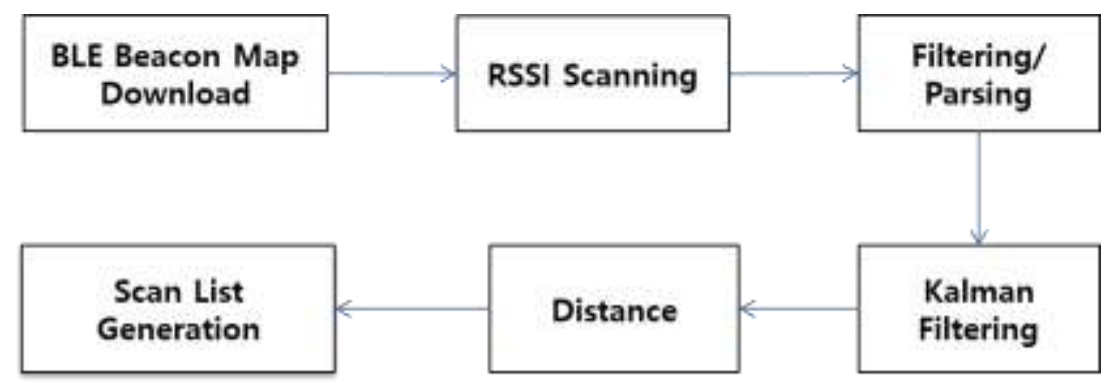

Figure 5. Scan List Generation Procedure

Fourth is weighing every scanned RSSI value according to strength layers and generating temporary coordinate(TC). As Figure 6 illustrates, when BLE RSSI is under $60 \mathrm{dBm},-60 \sim-70 \mathrm{dBm}$ and over $-70 \mathrm{dBm}$, we weigh 3, 2 and 1 times separately as Figure 6 left table. With weighed BLE beacons, mobile device temporary coordinates are generated as shown in the image on the right in Figure 6.

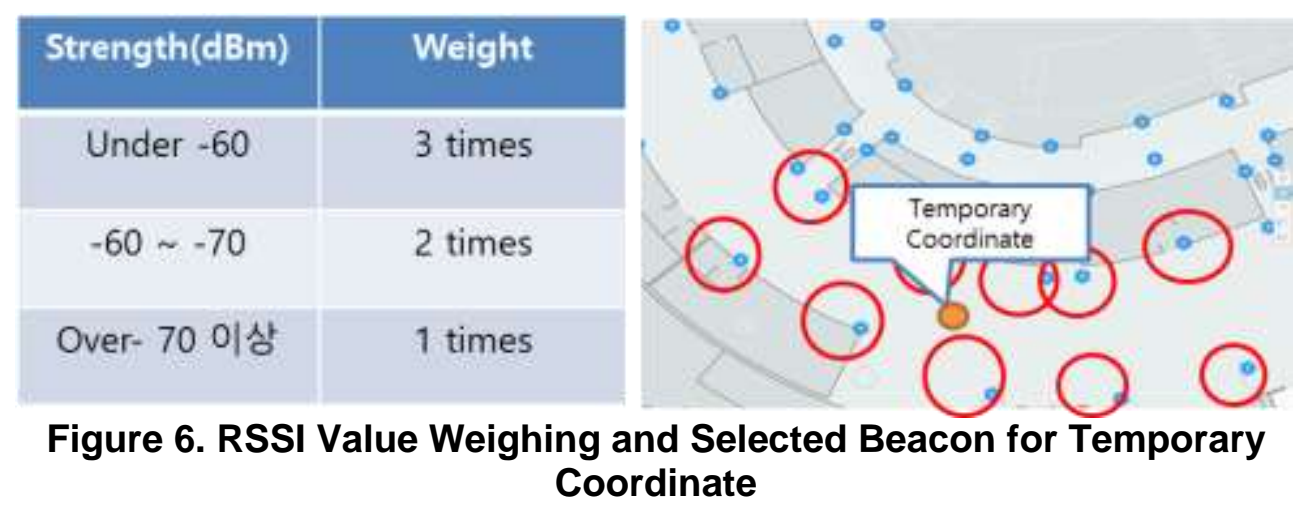


Fifth is cohesive analysis. By excluding beacons located too far from the calculated temporary coordinates of the mobile device, its accuracy is increased. At this time, the criterion for determining a distance depends on the local characteristics of the beacon. In this study, the distance standard is $20 \mathrm{~m}$ and the RSSI strength is higher than $-80 \mathrm{dBm}$. Figure 7 below shows red circled beacons that were chosen to increase the accuracy of device location after a cohesive analysis.
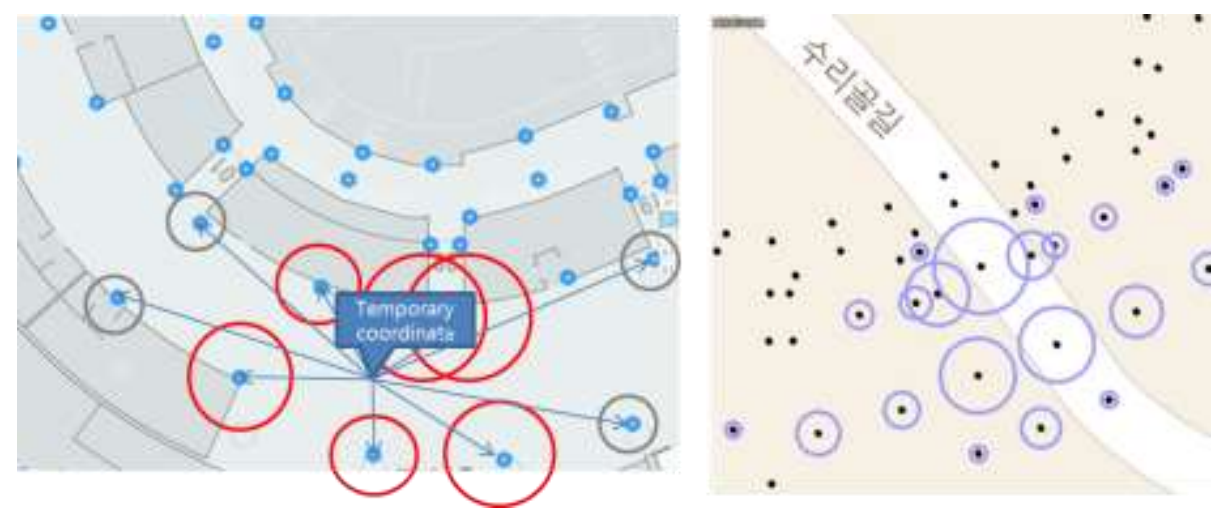

\section{Figure 7 Temporary Coordinate with Cohesive Analysis and After Attenuation Correction}

Sixth is the correction of attenuation. The coordinates of beacons are generated once per second. However, beacons scanned one second ago frequently are not scanned again. In addition, when a mobile device stays remains in the same place without moving, the same RSSIs are scanned continuously. We applied the attenuation logic to correct the problem to increase its cohesion, preventing the occurrence of outliers. The panel on the right in Figrue 7 above shows circled beacons after attenuation correction has been applied.

Seventh, a second weighting is applied to each scanned RSSI value to improve positioning accuracy. The result of all seven processes is referred to as the circle of positioning and will be used in combination with the Pedestrian Dead Reckoning(PDR) technique. The below described processes including the scanned list of RSSI values on scan list $1^{\text {st }}$ and $2^{\text {nd }}$ weighting of detected signals, temporary coordinate generation, cohesive analysis, removal of outlier BLE beacons, and attenuation adjustment are shown in below Figure 8. With the scan list, a positioning circle is generated every second, which will be used in combination with the PDR technique.

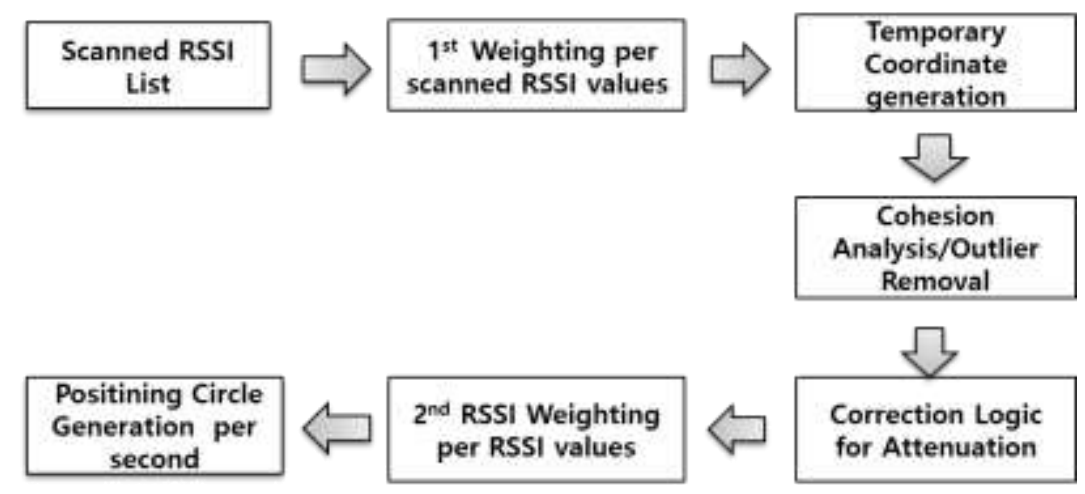

Figure 8. Positioning Circle Generation Procedures

After seven processes, we finally generate the positioning circles which have a fivemeter radius, which are the purple-colored circles shown in Figure 9 below; 


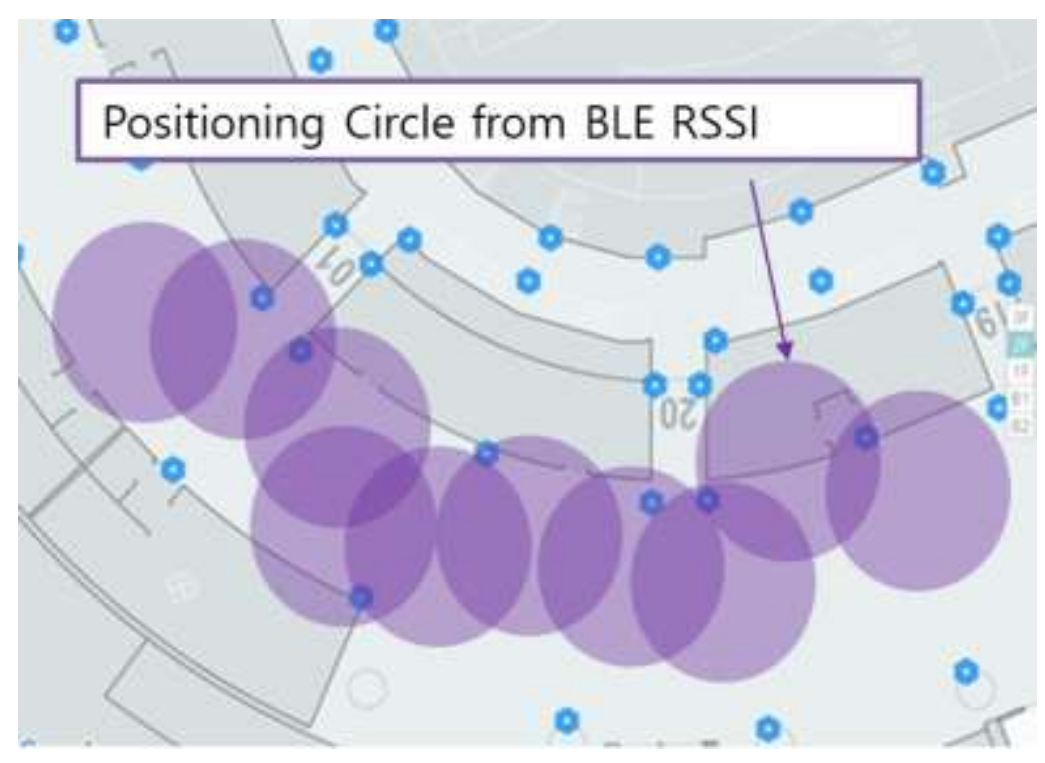

Figure 9. Positioning Circles

Positioning circles are criteria determining which PDR technique will be applied to find the location of a mobile device. When the device location calculated with IMU Sensors is outside of the purple circle, then the location will be adjusted into the circle.

Finally, Pedestrian Dead Reckoning(PDR) is applied. PDR is the process of calculating from a pedestrian's current position to a new one by using the previously determined position and advancing it based upon known or estimated speeds over an elapsed time and course. The PDR technique is naturally composed of 4-part estimation for step number, stride, heading and velocity. It is a technique to recognize the motion and situation of pedestrian or moving objects using an accelerometer, a gyroscope, a magnetometer and a barometer, which are Inertial Measurement Units(IMU) [9].

It is a self-contained system that measures the acceleration in the linear and angular motion such as direction, transverse direction and height and the angular velocity of roll, pitch and yaw. IMU sensors can integrate the acceleration and angular velocity obtained from the IMU sensors and calculate the pedestrian's speed and attitude angle This is possible because smartphones typically have a three-axis accelerometer, a three-axis angular speedometer, and a compass $[8,9]$.

Figure 10 shows the framework of a new estimation method which we developed with filtering sensor data to improve the accuracy of current PDR. Gyroscopes' angular rates can be integrated to time to calculate current orientation. Acceleration data from an accelerometer can be integrated into frame to calculate the moving speed or velocity. An accelerometer measures linear acceleration along its sensitive axis. The measurements from gyroscopes, accelerometer and magnetometer can be corrupted by noise, biases and other nonidealities, and the filtering process is integrated.

A Pedestrian's number of steps, length of step(stride) and heading are calculated with filtered sensor data to estimate the pedestrian's temporary coordinate which will be recalibrated to enhance coordinate accuracy. The coordinate is recalibrated so that the throughput of the integrated PDR frame combines with the BLE positioning circle. When estimated PDR coordinates are out of the BLE positioning circle, then those coordinates are adjusted and moved into the nearest circle. 


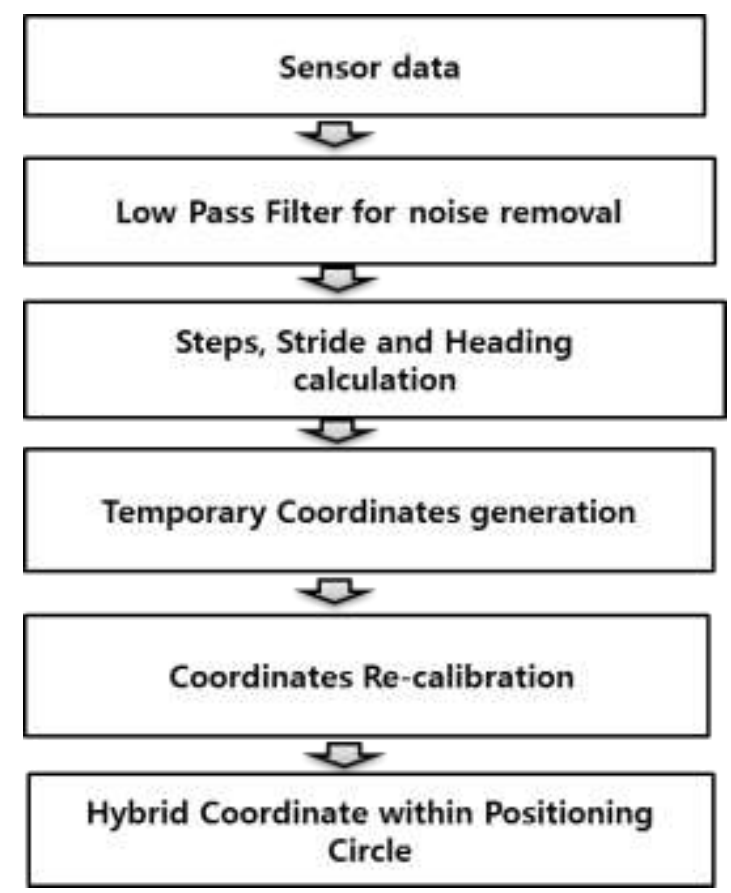

\section{Figure 10. Pedestrian Dead Reckoning Frame with Positioning Circle}

Figure 11 below illustrates that purple circles are BLE positioning ones and black or red dots are device coordinates from PDR estimation. When device coordinates locate in the circles, coordinates obtained from PDR location estimation are adapted: otherwise coordinates are out of circles, those coordinate positions are adjusted and coordinates moved into circles to prevent an accumulation error of typical problems of PDR. Figure 11 below shows PDR position adjustment, and the red-colored dots are moved into purple circles to correct the current position and moving path.

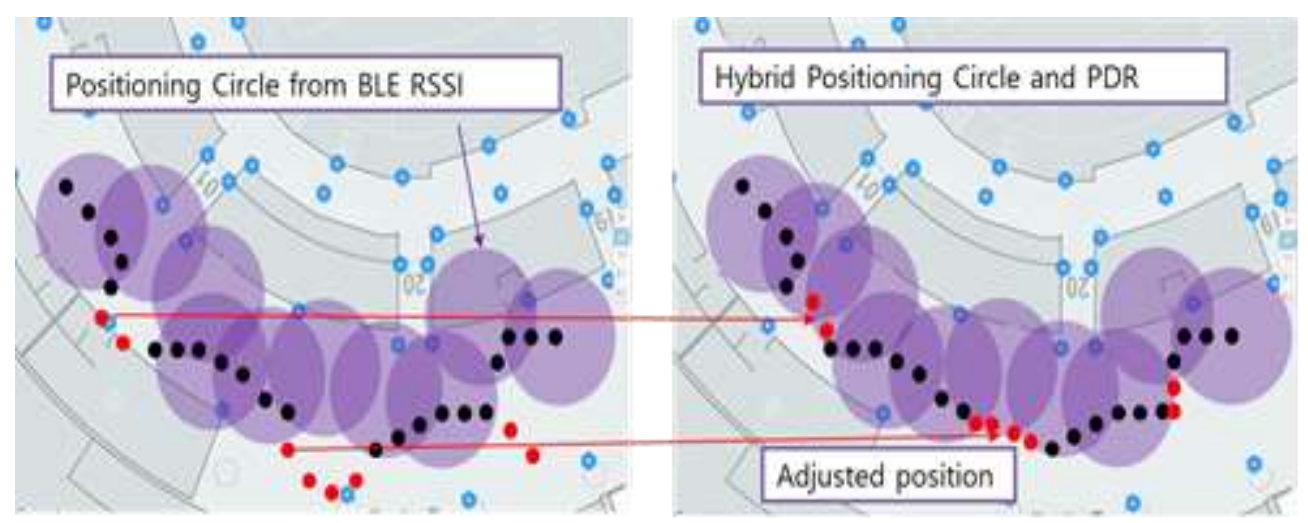

Figure 11. Adjustment PDR Coordinate within Positioning Circle

Figure 12 and Figure 13 show a comparison of BLE only and the hybrid of BLE positioning circle and PDR. In Figure 12, we can see a significant accuracy error with the BLE RSSI only method. But Figure 13 shows much more position accuracy than Figure 12. Figure 13 is the result of PDR combination with the BLE positioning circle algorithm. 


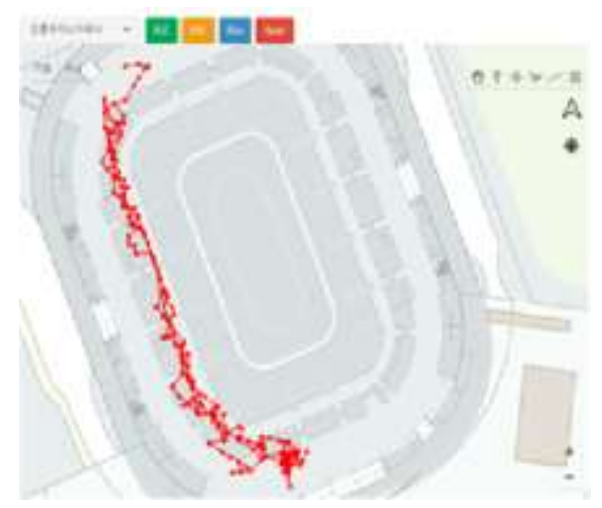

Figure 12. Before Hybrid Techniques Applied

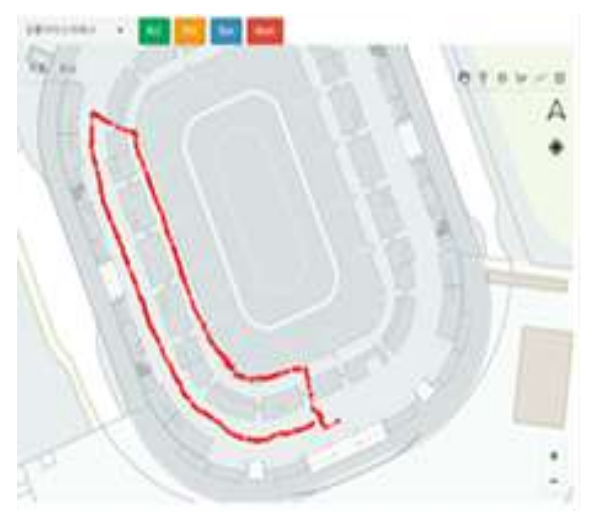

\section{Figure 13. BLE Positioning Circle and PDR Hybrid}

\section{Experiments Set Up}

Precision measurements were carried out using Android, and Apple smartphones in hockey arena which has various spaces and shapes and many communication barriers causing radio interference and propagation. The double floor space is 37,480 square meters, with 8,000 seats for spectators. An Eeectrical substation and building control center are installed under the rink, reducing the accuracy of the inertial measurement system.

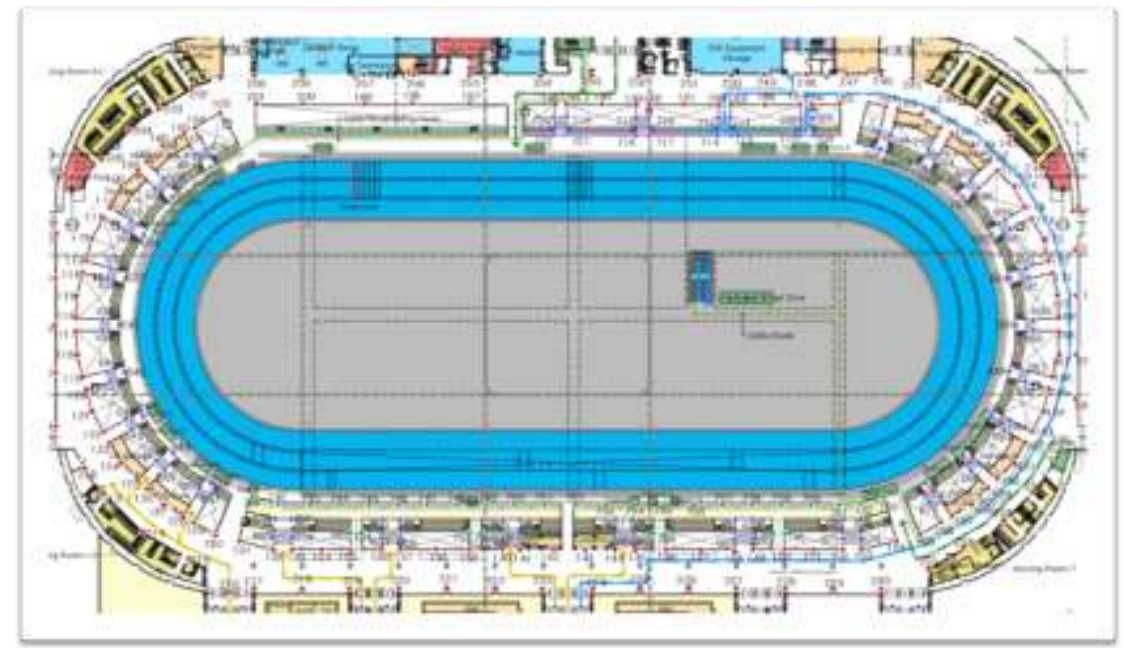

Figure 14. BLE Beacons Location Map 
112 beacons were installed at the spectator seating area on the $2^{\text {nd }}$ floor, and 235 were placed on walls and ceilings in corridors and crowded places on the $1^{\text {st }}$ floor. Beacon place map data is stored on the server as in the form of a database. Once the mobile device indoor positioning system activates, map data is automatically downloaded by mobile devices. Figure 14 above shows the location of BLE beacons, and each beacon has its own number.

Real-time distance and location calculations were performed on the mobile device at 50 chosen mostly busy locations including gates, corridors and busiest points. 50 access points were chosen as experiment points. Most busy and radio attenuation places were selected for testing under actual field condition.

\section{Experiment Results}

Figure 15 below shows the average data measured 10 times a day for 10 days - that is, a total of 100 times at 50 test points. The numbers represent distance error in meters between the actual and the estimated locations. The average distance error of BLE only is $6.0 \mathrm{~m}$ for 50 test points. Error numbers excessively larger than the average indicate the presence of communication obstacles such as columns, walls and many movements of the crowd which cause severe reflection, interference and attenuation of radio waves. However, at point 25, the error between actual and estimated position was reduced from $10.0 \mathrm{~m}$ to $1.4 \mathrm{~m}$ by applying the positioning circle and PDR hybrid algorithm. The results of the experiment using the BLE Beacon positioning circle and PDR hybrid algorithm show that the average error was are reduced from $6.0 \mathrm{~m}$ to $2.0 \mathrm{~m}$.

\begin{tabular}{|c|c|c|c|c|c|c|c|c|c|c|c|c|c|c|}
\hline Point & BLE & Hybarid & Point & BLE & Hybarid & Point & BLE & Hybrid & Point & BLE & Hybrid & Point & BLE & Hybrid \\
\hline 1 & 6.0 & 1.2 & 6 & 40 & 17 & 11 & 7.7 & 24 & 16 & 36 & 20 & 21 & 7.4 & 20 \\
\hline 2 & 5.2 & 1.8 & 7 & 39 & 12 & 12 & 63 & 3.9 & 17 & 3.7 & 0.9 & 22 & 5.9 & 2.7 \\
\hline 3 & 7.4 & 3.1 & 8 & 5.4 & 2.8 & 13 & 89 & 1.1 & 18 & 7.9 & 2.6 & 23 & 4.0 & 2.2 \\
\hline 4 & 6.1. & 2.4 & 9 & 55 & 2.2 & 14 & 50 & 29 & 19 & 67 & 2.5 & 24 & 80 & 1.3 \\
\hline 5 & 7.8 & 2.1 & 10 & 82 & 1.5 & 15 & 91 & 23 & 20 & 4.1 & 1.7 & 25 & 10.0 & 1.4 \\
\hline Peint & BLE & Hybrid & Point & SLE & Hybrid & Point & BitE & Hybrid & Point & BLE & Hybrid & Point & BLE & Hybrid \\
\hline 26 & 9.4 & 3.3 & 31 & 53 & 2.5 & 36 & 5.7 & 22 & 4: & 5.6 & 15 & 46 & 43 & 2.1 \\
\hline 27 & 3.2 & 2.1 & 32 & 83 & 3.8 & 37 & 91 & 15 & 42 & 4.6 & 2.1 & 47 & 7.1 & 2.1 \\
\hline 28 & 6.8 & 1.8 & 33 & 6.5 & 2.9 & 38 & 7.1 & 1.2 & 43 & 5.2 & 1.3 & 48 & 4.9 & 1.9 \\
\hline 29 & 4.1 & 1,1 & 34 & 61 & 23 & 39 & 49 & 26 & 44 & 6.1 & 1.7 & 49 & 5.1 & 1.8 \\
\hline 30 & 4.1 & 0.7 & 35 & 30 & 3.0 & 40 & 50 & 21 & 45 & 5.5 & 1.9 & 50 & 4.1 & 2.0 \\
\hline
\end{tabular}

Figure 15. Comparison of BLE Only and Hybrid Technique with Positioning Circle + PDR at 50 Experimental Points

The graph in Figure 16 shows an accuracy improvement of 65\% after applying the positioning circle and PDR hybrid technique at each of the 50 experimental points. The average error was reduced from $6.0 \mathrm{~m}$ to $2.0 \mathrm{~m}$ at 50 places, a $65 \%$ improvement.

Average distance error was determined to be $2.0 \mathrm{~m}$ in a very large two-story sports facility which accommodated over 8,000 spectators. 


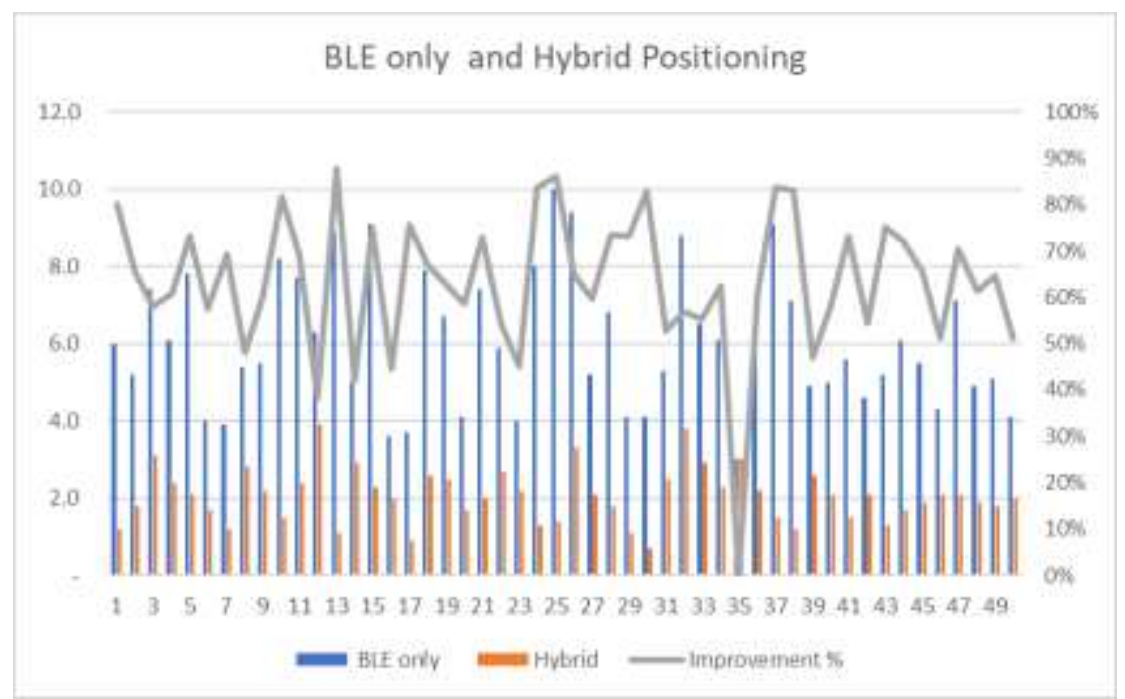

\section{Figure 16. Location Estimation Errors between BLE Only and Hybrid Techniques}

\section{Conclusion}

In this paper we propose a BLE Positioning circle and PDR hybrid method to improve the accuracy of indoor positioning. The results of the study show that the average error of the direction and the moving distance in indoor environment is within $2.0 \mathrm{~m}$, making it suitable for indoor positioning systems or indoor navigation for ultra-large spaces, such as airports and sport stadiums. The indoor positioning technique proposed in this study can be used as seamless navigation by combining it with GPS and Wi-Fi for outdoor positioning, and can also be applied to Augmented Reality.

\section{References}

[1] Z. Li, C. Liu, J. Gao and X. Li, "An Improved WiFi/PDR Integrated System Using an Adaptive and Robust Filter for Indoor Localization", Internal Journal of Geo-Information, ISPRS Int. J. Geo-Inf., (2017).

[2] T. Heinecke and M. Wolfe, "The Role of Bluetooth Low Energy for Indoor Positioning Application", Computer Science Department, Montana State University, Bozeman, MT USA.

[3] X. Li, J. Wang and C. Lui, “A Bluetooth/PDR Integration Algorithm for an Indoor Positioning System”, Sensors (Basel), vol. 15, no. 10, (2015) October.

[4] K. Kaemarungsi and P. Krushnamurthy, "Modeling of Indoor Positioning System Based on Location Fingerprinting", IEEE, Telecommunication Program, School of Information Science, University of Pittsburgh, Pennsylvania, (2004).

[5] J. Huh, C. Lee and J. Kim, "A study of beacon delivery characteristics in BLE based fingerprinting indoor positioning system", in proc. KIISE, vol. 2015, no. 6, (2015), pp. 1612-1614.

[6] R. Faragher and R. Harle, "Innovation: Getting Closer to Everywhere", GPS World, (2013) October.

[7] S. Chai, R. An and Z. Du, "And Indoor Algorithm Using Bluetooth Low Energy RSSI", AMSEE, (2016).

[8] R. Faragher and R. Harle, "Innovation: Getting Closer to Everywhere", GPS World, (2013) October.

[9] L. Fang, P. J. Antsaklis, L. A. Montestruque, M. Brett McMickell, M. Lemmon, Y. Sun, H. Fang, I. Koutroulis, M. Haenggi, M. Xie and X. Xie, "Design of a Wireless Assisted Pedestrian Dead Reckoning System-The NavMote Experience", IEEE Transactions on Instrumentation and Measurement, vol. 54, no. 6, (2005) December.

[10] S.-J. Jeong and J.-H. Yim, "Implementation of the Passenger Positioning Systems using Beacon", The Korea Institute of Information and Communication Engineering, Journal of the Korea Institute of Information and Communication Engineering, vol. 20, no. 1, (2016) January, pp. 153-160.

[11] Y.-I. Kim, M. Kim and H.-C. Kim, "Implementation of total management system for exhibitions and Convention using beacon", Asia-pacific Journal of Multimedia Services Convergent with Art, Humanities, and Sociology, ISSN:2383-5281, vol. 6, no. 2, (2016) February, pp. 35-44, http://dx.doi.org/10.14257/AJMAHS.2016.19. 


\section{Authors}

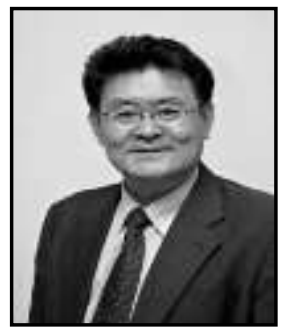

Hee-Yong Kang, received his Master's degree in Business Administration from Korea University, Seoul (1994) and his master's degree in Science (1999), and doctoral degree in IT Policy and Management in Soongsil University, Seoul (2015). His research interests are focued on the Internet of Things and Bigdata.

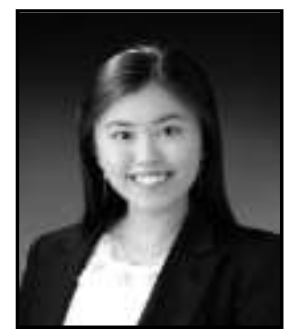

Ji-Na Lee, she received her bachelor's degree of computer science in Dankook University, Seoul (2008). Her current research interest includes Internet of Things, Bigdata.

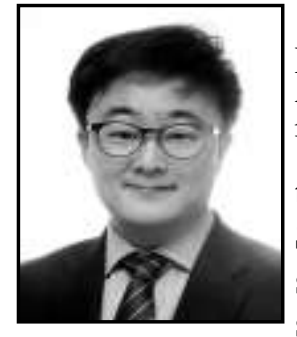

Hyungwoo Park, received a Ph.D., an M.S., and a B.S. in Electrical Engineering from Soongsil University. He is an assistant professor at the Information Technology Department in Soongsil University, Seoul, Korea. He is studied railway noise reduction technology at Korea Railroad Research Institute prior to joining Soongsil University. His current research interests include sound signal processing, big data analysis, voice analysis, noise reduction system, wave field synthesis, railway noise, and Internet of Things.

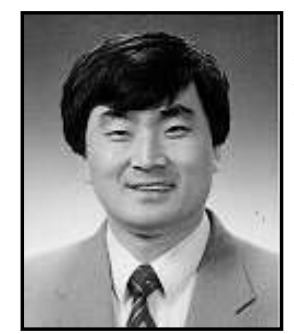

Myung-Jin Bae, received the Ph.D. degree in Electronic Engineering from Seoul National University in 1987. He is currently the Professor of the Dept. of Information \& Telecommunication at Soongsil University, Seoul, Korea.

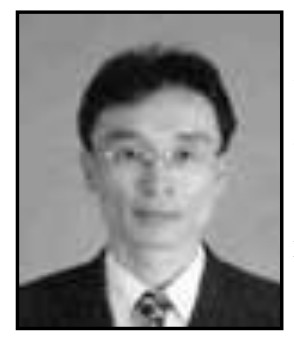

Jong-Bae Kim, received his bachelor's degree in Business Administration from University of Seoul, Seoul (1995), his master's degree (2002), doctoral degree of Computer Science from Soongsil University, Seoul (2006). He is currently a professor at the Graduate School of Software in Soongsil University, Seoul, Korea. His research interests are focused on Software Engineering, and Open Source Software. 
International Journal of Control and Automation

Vol. 11, No. 8 (2018) 